\title{
Review Article \\ Core-Shell Nanocatalysts Obtained in Reverse Micelles: Structural and Kinetic Aspects
}

\author{
Concha Tojo, ${ }^{1}$ David Buceta, ${ }^{2}$ and M. Arturo López-Quintela ${ }^{2}$ \\ ${ }^{1}$ Physical Chemistry Department, University of Vigo, 36310 Vigo, Spain \\ ${ }^{2}$ Laboratory of Magnetism and Nanotechnology, University of Santiago de Compostela, 15782 Santiago de Compostela, Spain \\ Correspondence should be addressed to Concha Tojo; ctojo@uvigo.es
}

Received 15 May 2015; Accepted 15 September 2015

Academic Editor: Xianwen Zhang

Copyright (c) 2015 Concha Tojo et al. This is an open access article distributed under the Creative Commons Attribution License, which permits unrestricted use, distribution, and reproduction in any medium, provided the original work is properly cited.

\begin{abstract}
Ability to control the metal arrangement in bimetallic nanocatalysts is the key to improving their catalytic activity. To investigate how metal distribution in nanostructures can be modified, we developed a computer simulation model on the synthesis of bimetallic nanoparticles obtained in microemulsions by a one-pot method. The calculations allow predicting the metal arrangement in nanoparticle under different experimental conditions. We present results for two couples of metals, Au/Pt $(\Delta \varepsilon=0.26 \mathrm{~V})$ and $\mathrm{Au} / \mathrm{Ag}(\Delta \varepsilon=0.19 \mathrm{~V})$, but conclusions can be generalized to other bimetallic pairs with similar difference in standard reduction potentials. It was proved that both surface and interior compositions can be controlled at nanometer resolution easily by changing the initial reactant concentration inside micelles. Kinetic analysis demonstrates that the confinement of reactants inside micelles has a strong effect on the reaction rates of the metal precursors. As a result, the final nanocatalyst shows a more mixed core and a better defined shell as concentration is higher.
\end{abstract}

\section{Introduction}

The development of new improved nanocatalysts has attracted extensive research due to scientific and technological interest [1-3]. Special attention was paid to core-shell bimetallic nanoparticles. The presence of an additional metal in bimetallic nanoparticles leads to different properties from monometallic ones. Such particles not only maximize the catalytic surface due to a high surface-to-volume ratio, but also enhance catalytic properties in comparison to monometallic nanoparticles even at lower temperatures $[4,5]$. Specifically, the catalytic behaviour of monolayers of one metal on another metal was shown to be definitely different compared to those of the parent metals [6]. It was shown that the formation of a bond between two different metals on a surface induces an electronic transfer towards the metal with more empty states in the valence band. This implies that the heteronuclear metal-metal bond depends on the geometry of the bimetallic particle [7], which in turn strongly affects catalytic activity. As an example, the catalytic activity of $\mathrm{Au} / \mathrm{Pt}$ particles was shown to be directly related to surface composition [8]. Therefore, the ability to control the metal arrangement in bimetallic nanoparticles is the key to improving the catalytic activity.

In spite of existing several successful strategies to obtain core-shell bimetallic nanoparticles, the control of the metals arrangement is still an open question [8-11]. Moreover, the metal distribution in a bimetallic nanoparticle depends not only on the particular couple of metals but also on the preparation method $[12,13]$. So we will focus our attention on one particular route to prepare bimetallic nanoparticles, the precipitation of nanoparticles inside the droplets of a microemulsion $[9,10,14,15]$. A water in oil (w/o) microemulsion consists of water droplets dispersed in the oil phase and stabilized interfacially by a surfactant monolayer [16, 17]. Reactants (metal precursors and reducing agent) are solved inside the water droplets. Mixing of two or more microemulsions (each containing one of the reactants) can result in the formation of monodisperse metal particles inside these aqueous nanoreactors. The reacting species are believed to be exchanged between water droplets during interdroplet collision, which can be able to open a channel for mass 
transfer $[14,18]$. Microemulsion micelles are ideal templates because each droplet can isolate a particle from those in neighboring droplets. In this way, particle aggregation is prevented, so this method allows a precise control over the composition and size of the final nanoparticles. In the case of bimetallic nanoparticles, the reacting species are two different metal precursors and a reducing agent, initially located in different microemulsion droplets. Due to collisions and material interdroplet exchange, both chemical reductions can take place inside the same water pool, resulting in a bimetallic nanoparticle which remains confined in the micelle. Nanoparticles composed of different couples of metals have been prepared by this method [19-22]. Nevertheless, even using microemulsions, there are a high number of variables controlling the final arrangement of the two metals in the nanoparticle. In order to optimize the metal distribution in a bimetallic nanocatalyst, a deeper understanding of the mechanism of nanoparticle formation in microemulsions is needed. We have developed a Monte Carlo simulation model, which predicts the metal distribution in the bimetallic particle and allowed us to study nanoparticle formation from a kinetic point of view. The validity of the model predictions was proved by comparison between experimental and simulation results [23]. The metal arrangement of $\mathrm{Au} / \mathrm{Pt}$ nanoparticles prepared in a $75 \%$ isooctane $/ 20 \%$ tergitol $/ 5 \%$ water microemulsion was studied by HR-STEM (scanning transmission electron microscopy) and cross section with EDX analysis was performed to get their nanostructure. Theoretical STEM profiles were calculated from the structures predicted by simulation, using the same synthesis conditions compared to those of experimental studies. A successful concordance between simulated and experimental STEM profiles supports the validity of the simulation model.

For a better understanding of the metals arrangement in a bimetallic nanocatalyst, one must take into account that a nanoparticle is formed from a nucleus, which can grow by building up new layers as new atoms are deposited on the previous ones. If there are two different metal atoms in the reaction medium, the metal distribution in final particle will be defined by the sequence of deposition of the two metals. The long term objective of the present study is implementing the know-how in the preparation of bimetallic nanoparticles in microemulsions. Such a challenge aim can only be reached if the factors affecting the sequence of metals are analyzed.

First, it is well-known that this sequence is conditioned by the rates of chemical reduction of the two metal ions. If ions of two different metals are very close to each other (as inside a water pool), and one metal reduces faster than the other, the sequence of deposition will be first the faster metal and then the slower one. So the resulting distribution will be a core-shell structure. On the contrary, a mix of metals is expected when both ions are reduced simultaneosuly. Therefore, at first, the key factor to decide what structure should be (core-shell or mix) is the difference in reduction potentials of both metals [1,24-26]. Nevertheless, it is not the only factor, because core-shell geometries have been obtained from metals with quite similar reduction potential, such as $\mathrm{Pt} / \mathrm{Ag}$ [27, 28], Pd/Au [29], and Pt/Pd [19, 20, 30-32].
Second, the order of metals deposition is also affected by the nucleation rate of each metal $[33,34]$. This is based on the fact that clustering used to take a longer time than reduction [35], so the chemical reduction is usually considered as instantaneous. In this case, the subsequent nucleation process would be the major factor to determine the order of metals. Consequently, the majority of seeds from which nanoparticles are formed will be composed of the metal with the fastest nucleation. The growth of these seeds will lead to a monometallic core. The slower metal would be deposited on the core forming the surrounding shell. This argument was used to explain Pd-Pt bimetallic nanocluster structures $[20,21,36]$.

Also relevant to the discussion is the observation that the last two factors, reduction rate and nucleation rate, are characteristics of the particular couple of metals. That is, if one wants to prepare a bimetallic nanoparticle composed of two specific metals, the reduction potentials and the critical nucleus sizes cannot be modified. However, both processes (reduction and nucleation) can be altered by changing the values of reactants concentration. Therefore, a third factor affecting the sequence of metals can be concentration, which is easily modified.

Finally, one must keep in mind that micelles are demonstrated to be a reaction medium that does not always obey classical assumptions. As an example, it was proved that the usual definition of $\mathrm{pH}$ cannot be employed inside micelles [37]. So chemical kinetics in micelles must take into account the compartmentalization of the reaction media [38]. Although the fact that reactants are trapped into micelles plays a key role in metal arrangement in bimetallic nanoparticles, studies in this direction are limited. In the research at hand, a comprehensive analysis of the resulting nanostructures is performed, based on the hypothesis that metal arrangement is due to the particular combination of three main factors: the difference in reduction rate of both metals (characteristic of the pair of metals), the intermicellar exchange rate (determined by microemulsion composition), and the amount of metal precursors inside micelles. All together give rise to a particular order of metals deposition, which in turn determines the metal distribution in final nanoparticle. To investigate how metal distribution in the nanostructure can be easily modified by changing reactant concentration, two couples of metals were chosen, and microemulsion composition was kept unvariable. Because of their high catalytic activity the nanocatalysts chosen for the study were $\mathrm{Au} / \mathrm{Pt}[12,13,39-42]$ and $\mathrm{Au} / \mathrm{Ag}$ [43-46]. The couple of the metals was characterized by the ratio between the reduction rates of the fast and the slow metal. The structure of $\mathrm{Au} / \mathrm{Pt}$ nanoparticles (difference in standard reduction potentials $\Delta \varepsilon=0.26 \mathrm{~V}$ ) was shown to be successfully reproduced by simulation by means of reduction rates ratio $v_{\mathrm{Au}} / v_{\mathrm{Pt}} \approx 10$ [23]. By means of the Volmer equation, a smaller difference in standard reduction potentials such as $\mathrm{Au} / \mathrm{Ag} \operatorname{system}(\Delta \varepsilon \approx$ $0.19 \mathrm{~V})$ can be simulated by a reduction rate ratio $v_{\mathrm{Au}} / v_{\mathrm{Ag}} \approx 5$. Because the reduction rate ratio is the simulation parameter used to characterize the nature of the metals, all results can be generalized to other bimetallic systems whose difference in standard reduction potentials is about this range. 


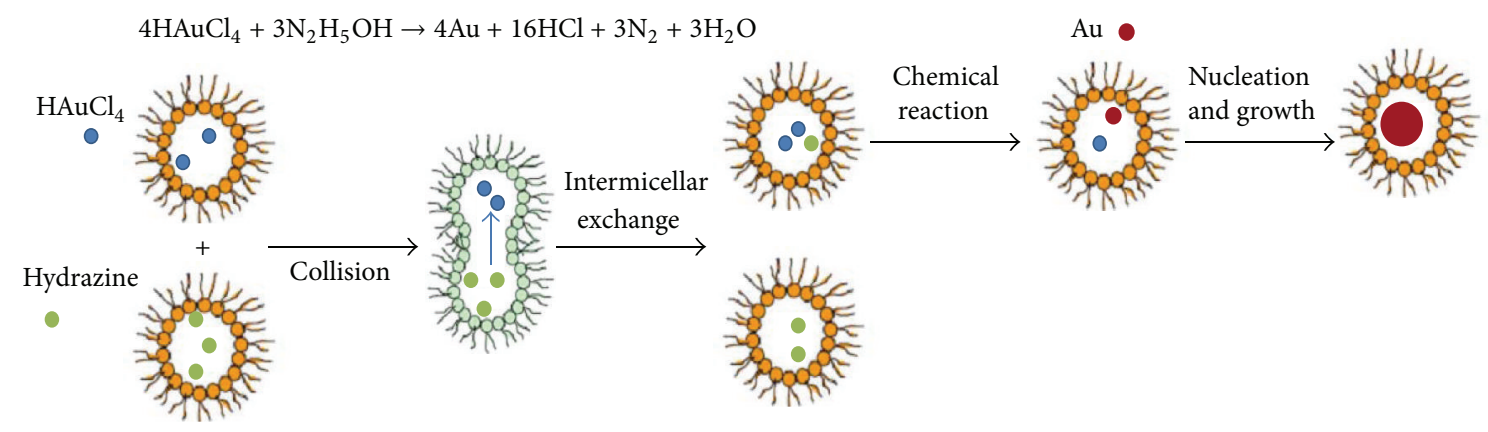

SCHEME 1: Scheme of nanoparticle formation inside microemulsion droplets.

\section{Simulation Model}

The kinetic course of the reaction is simulated as follows (see [47] for details): the microemulsion is described as a set of micelles, which move and collide with each other. Initially, each kind of reactant is located in one microemulsion. The reactants are the metal salts $\left(\mathrm{HAuCl}_{4}\right.$ and $\mathrm{H}_{2} \mathrm{PtCl}_{6}$ to prepare $\mathrm{Au} / \mathrm{Pt}$, or $\mathrm{HAuCl}_{4}$ and $\mathrm{AgNO}_{3}$ to prepare $\mathrm{Au} / \mathrm{Ag}$ ) and the reductor agent hydrazine. The concentration of reactants can be modified by varying the concentration of the water phase for each microemulsion. To study the influence of concentration we present results using different average values of the number of reactants inside the droplets, keeping a 1:1 proportion: $\left\langle c_{\text {Au salt }}\right\rangle=\left\langle c_{\mathrm{Pt} \text { or Ag salt }}\right\rangle=4,32,64$, and 128 metal ions in a droplet, which corresponds to $0.02 \mathrm{M}, 0.16 \mathrm{M}$, $0.40 \mathrm{M}$, and $0.64 \mathrm{M}$, respectively. Then microemulsions, each one containing one reactant, are mixed.

The diffusion of micelles is assumed to be governed by Brownian motion. Upon collision micelles can establish a water channel forming a transient dimer, exchanging their contents (reactants, products, and/or growing particles). In each step, $10 \%$ of micelles are randomly chosen to collide, fuse, and redisperse, allowing material exchange (see Scheme 1). One Monte Carlo step (mcs) is completed when the composition of colliding droplets is revised after collision according to the criteria described below.

The intermicellar exchange protocol of free units (reactants and nonaggregated metal atoms) consists in their redistribution between two colliding droplets in accordance with the concentration gradient principle: reactants and free metal atoms are transferred from the more to the less occupied droplet. The exchange parameter $\left(k_{\mathrm{ex}}\right)$ quantifies the maximum amount of reactants (metal precursors and/or reductor agent) and metallic atoms which can be transferred during a collision. When collision takes place between two micelles containing different kind of reactants, redistribution of material can lead to one metal precursor and the reductor being located inside the same micelle. At this stage, chemical reduction can take place. Model assumes that chemical reduction of $\mathrm{Au}$ precursor is instantaneous $\left(100 \%\right.$ of $\mathrm{HAuCl}_{4}$ inside the same micelle is reduced); that is, reaction is completed, and only Au atoms and excess of reactants (either $\mathrm{Au}$ salt and/or hydrazine) are to be distributed in daughter micelles. This implies that reactants Au salt and hydrazine will not exist together in a micelle. To take into account different reductions rates, the probability that reactants are trapped together in the same micelle which reduce to metal atoms can be diminished. To simulate the $\mathrm{Au} / \mathrm{Pt}$ synthesis only $10 \%$ of $\mathrm{H}_{2} \mathrm{PtCl}_{6}$ is reduced in each collision $\left(v_{\mathrm{Au}} / v_{\mathrm{Pt}}=10\right)$. In the case of $\mathrm{Au} / \mathrm{Ag}, 20 \%$ of the pairs $\mathrm{AgNO}_{3}$ and hydrazine available in the micelle produce $\mathrm{Ag}$ atoms $\left(v_{\mathrm{Au}} / v_{\mathrm{Ag}}=5\right)$. The rest of metal salt and reducing agent remain in the micelle and will be exchanged or will react in a posterior collision. All atoms produced in each micelle are considered to be aggregated forming a growing nanoparticle. As the synthesis advances, more micelles carry reactants and particles simultaneously. If one colliding droplet is carrying a particle, the reaction always proceeds on it, acting as nucleation point. When both colliding micelles contain particles, reaction takes place in the micelle containing the larger one, which has a larger surface, increasing the probability of playing as catalyst.

The intermicellar exchange protocol of growing particles is limited by the size of the channel through which colliding micelles exchange their content, which is determined by the flexibility of the surfactant film. The flexibility parameter $(f)$ specifies the maximum particle size for transfer between micelles. The exchange criteria of particles are also dictated by Ostwald ripening, which assumes that the largest particles will grow by condensation of material, coming from the smallest particles that solubilise more readily than larger ones. Therefore, if both colliding micelles contain particle, the smaller one is transferred to the micelle carrying the larger one, whenever the channel size would be large enough.

Despite their simplicity, material intermicellar exchange protocols allow us to simulate the surfactant film flexibility. At first, the requirements for material intermicellar exchange to take place are that colliding micelles must remain long enough together, and the size of the channel connecting both micelles must be large enough. It can be assumed that the main factor determining the exchange of isolated species (reactants and free metals) is the dimer stability, because they traverse the intermicellar channel one by one. That is, more species can be exchanged when the two micelles stay together longer (higher dimer stability), and channel size would not be relevant. Based on this, $k_{\text {ex }}$ is related to the dimer stability. On the contrary, the channel size becomes decisive when the transferred material is a particle constituted by aggregation of metal atoms, which have to be exchanged as a whole. As a 
consequence, this kind of material exchange will be restricted by the intermicellar channel size ( $f$ parameter). From this picture, the flexibility of the surfactant film is included in the simulation model by means of $k_{\mathrm{ex}}$ (dimer stability) and $f$ (intermicellar channel size) parameters [48]. Good agreement between simulation and experimental results was obtained when a rigid film, such as AOT (dioctyl sodium sulfosuccinate)/n-heptane/water microemulsion, was related to a channel size $f=5$, associated with $k_{\text {ex }}=1$ free atom exchanged during a collision [49]. In case of flexible film, both factors have to rise together. In this study we present results obtained using a more flexible microemulsion, such as isooctane/tergitol/water, which was successful compared to simulation data using $f=30, k_{\mathrm{ex}}=5$ [23].

Each simulation run ends when the contents of each micelle remain invariable; that is, all metal salts were reduced and no material intermicellar exchange is taking place. It results in a set of micelles, each one of which can contain one particle. The metal distribution in the final particle is defined by the order with which the two metals are deposited on particle surface. Therefore, in order to study the particle composition, the sequence of the two metals in each micelle is monitored as a function on time.

The metals in each nanoparticle are assumed to take a spherical arrangement. Each nanoparticle sequence is divided in ten concentric layers, and the dispersity and averaged composition (\% $\mathrm{Au}$ ) are calculated layer by layer. Finally results are averaged over 1000 runs. The composition (\% Au) of each layer is showed by a colour grading: the degradation goes from blue $(0 \%-10 \%$ of fast metal $\mathrm{Au})$ to red $(90 \%-100 \% \mathrm{Au})$. Grey means a $50 \%$ of each metal. A lighter colour indicates a higher proportion of pure metal in the layer. The resulting structures are represented as histograms, in which the number of particles with a given $\% \mathrm{Au}$ is monitored layer by layer. In this way the layer composition can be observed from the core (inner layer) to the shell (outer layer). It allows us to study how the metal arrangement changes as the synthesis advances. Likewise, the nanoparticle composition is also described by means of concentric spheres. The thickness of each sphere is proportional to the number of layers with a given $\% \mathrm{Au}$, keeping the same colour code.

\section{Results and Discussion}

3.1. Changing the Metal Salt Concentration: Structural Study on Metals Distribution in Final Nanoparticle. First of all, we will study the metal distribution in final nanoparticle for two different values of the reduction rates ratio of the two metals. To isolate this dependence, the microemulsion composition (characterized by $f=30, k_{\mathrm{ex}}=5$ ) and the nucleation rates of both metals $\left(n_{\mathrm{Au}}^{*}=n_{\mathrm{Ag}}^{*}=n_{\mathrm{Pt}}^{*}=1\right)$ must be kept constant. Figure 1 shows the predicted structures. In these figures, the reduction rate of the fast metal $(\mathrm{Au})$ is considered instantaneous: that is, $100 \% \mathrm{Au}$ salts inside the same micelle give rise to $\mathrm{Au}$. The reduction rate of the slower metal (Pt or $\mathrm{Ag})$ is decreased from $v_{\mathrm{Au}} / v_{\mathrm{Ag}}=5(20 \% \mathrm{Ag}$ salts react in each Monte Carlo step; see first column in Figure 1) to $v_{\mathrm{Au}} / v_{\mathrm{Pt}}=10$ (10\% Pt salts react; see second column in Figure 1). Each file in Figure 1 shows results for different values of initial metal salts concentration.

Figure 1(a) shows the nanostructure obtained using a small difference in reduction rates and a low concentration $\left(v_{\mathrm{Au}} / v_{\mathrm{Pt}}=5,\langle c\rangle=4\right.$ reactants per micelle). One can see that the composition of the inner layers (core) is enriched in the faster reduction metal ( $\mathrm{Au}$, see red bar on the left). By observing from the inner to the outer layers, the composition of the surrounded layers evolves to a mix of the two metals as the synthesis advances. At the end of the process, most of the particles have a shell which is composed of approximately $50 \%$ in each metal. When the reduction rate ratio is larger (see Figure $1(\mathrm{~b}), v_{\mathrm{Au}} / v_{\mathrm{Pt}}=10,\langle c\rangle=4$ ), more Au is mainly located in the core, and the surrounding shells show a slight enrichment in the slower metal. It was shown that a further increase in $v_{\text {fast }} / v_{\text {slow }}$ gives rise to better segregated metals $[22,25,26]$. In contrast, a further decrease in reduction rate ratios leads to more mixed structures, so an alloy was obtained for equal reduction rates $\left(v_{\text {fast }} / v_{\text {slow }}=1\right)$ [25]. From these results, one could think that the ratios between both reduction rates $v_{\text {fast }} / v_{\text {slow }}=5,10$ are not large enough to give rise to a core-shell distribution. However, if concentration is increased (keeping constant the reduction rate ratio) the metal distribution changes drastically: most of the particles show an Au core, surrounded by mixed middle layers, finally covered by a slow metal-shell (see blue bar on the right in Figure 1). Two main features must be pointed out from this figure: First, independently of reactants concentration, the metal segregation is better as the reduction rate ratio is larger (see Figure 1, from left to right), as expected. Second, nanostructure can be modified by a change in concentration, as experimentally observed [23]. To provide a deeper insight into the influence of the initial reactant concentration on metal distribution, histograms in Figure 1 must be compared from the top to the bottom. The first column in Figure 1 clearly shows how, in spite of the small difference in chemical reduction rate ratio, the metal segregation is enhanced by high reactant concentration: from an Au-enriched core covered by an alloyed shell obtained at small concentration (see Figure 1(a), $\langle c\rangle=4$ metal ions in a micelle) to the incipient formation of a shell at higher concentration (see Figure $1(\mathrm{c}),\langle c\rangle=32$ ), which evolves to a better defined shell when concentration is increased (see blue bars on the right in Figure 1(e), $\langle c\rangle=$ 128). The same qualitative behaviour is obtained at larger reduction rate ratio (see second column in Figure 1). One can conclude that the metal distribution in a bimetallic nanoparticle can be modified at nanometer resolution just by changing the reactants concentration. This is an unexpected result, which is supported by experimental observations [23]. It is well-known that the rate of a bimolecular reaction, such as reduction, increases with concentration. But the concentration of both metal salts was the same in all cases, so it may be expected that the influence of concentration on both reductions was similar. However, the analysis of the histograms proves that metal segregation in core-shell nanostructures depends on concentration. On the one hand, the shell is more pronounced at higher concentration. On the other hand, concerning the core, note that the larger the concentration, the larger the quantity of the slow metal 


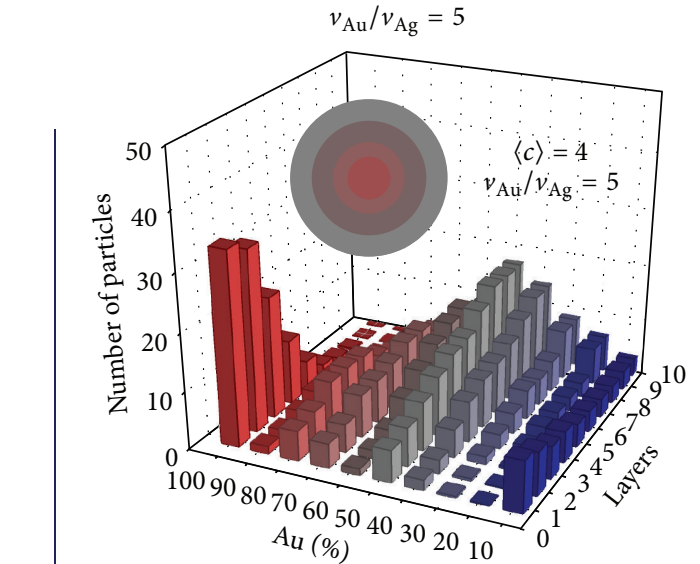

(a)

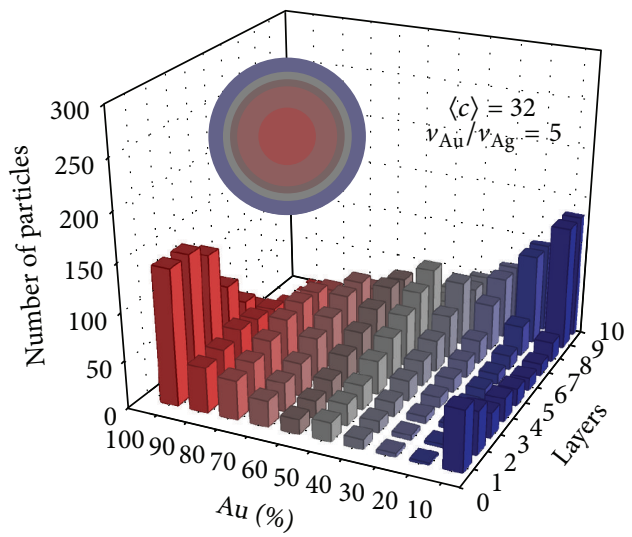

(c)

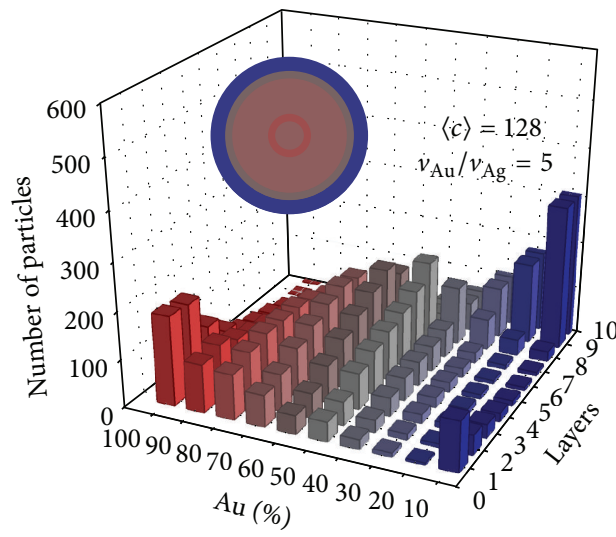

(e)

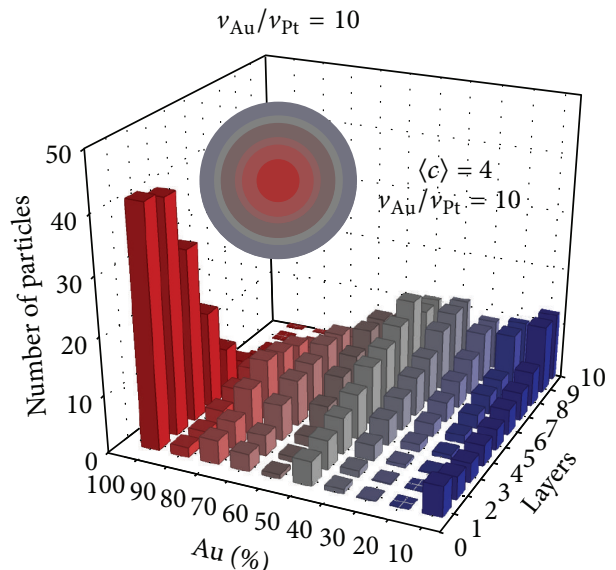

(b)

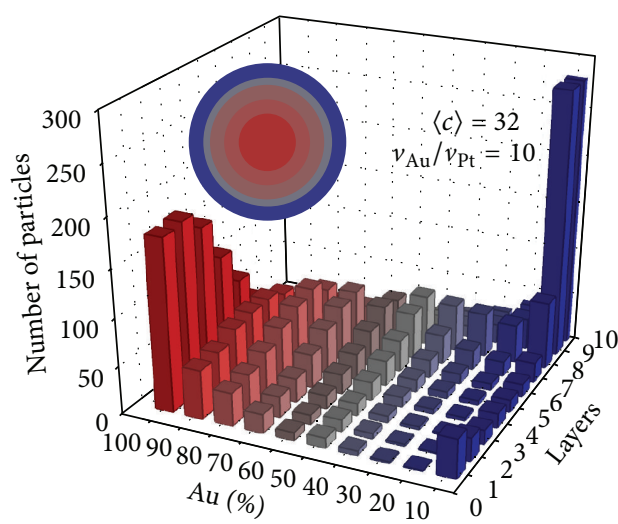

(d)

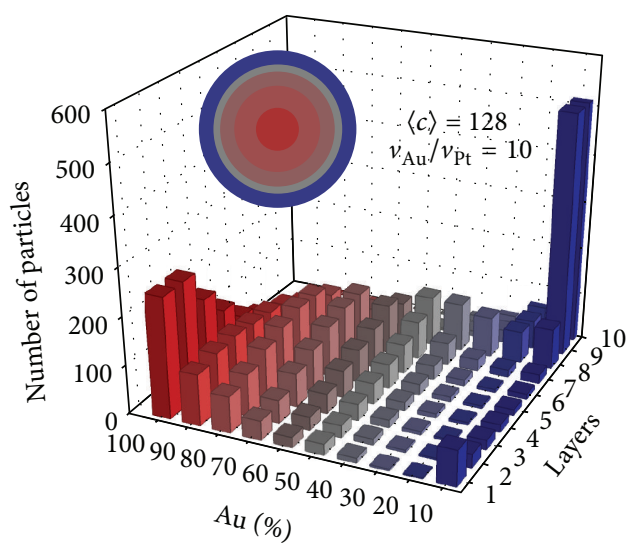

(f)

FIGURE 1: Histograms represent the number of particles with a given percentage of the faster metal (Au) in each layer, from the nanoparticle core to the surface for two different couples of metals. (a) Reduction rate ratio $v_{\mathrm{Au}} / v_{\mathrm{Ag}}=5$ and metal salt concentration $\langle c\rangle=4$ metal ions in a micelle; (b) reduction rate ratio $v_{\mathrm{Au}} / v_{\mathrm{Pt}}=10$ and concentration $\langle c\rangle=4$ ions; (c) $v_{\mathrm{Au}} / v_{\mathrm{Ag}}=5$ and $\langle c\rangle=32$; (d) $v_{\mathrm{Au}} / v_{\mathrm{Pt}}=10$ and $\langle c\rangle=32$; (e) $v_{\mathrm{Au}} / v_{\mathrm{Ag}}=5$ and $\langle c\rangle=128$; (f) $v_{\mathrm{Au}} / v_{\mathrm{Pt}}=10$ and $\langle c\rangle=128$ ions. Scheme color: blue ( $0 \%-45 \%$ of Au), grey ( $45 \%-55 \%$ of Au), and red $(55 \%-100 \%$ of $\mathrm{Au}$ ). Less red means less $\mathrm{Au}$ (faster metal). Circles in each histogram represent nanoparticle structure in concentric layers, keeping the same color scheme. Surfactant film flexibility $\left(k_{\mathrm{ex}}=5, f=30\right)$. 


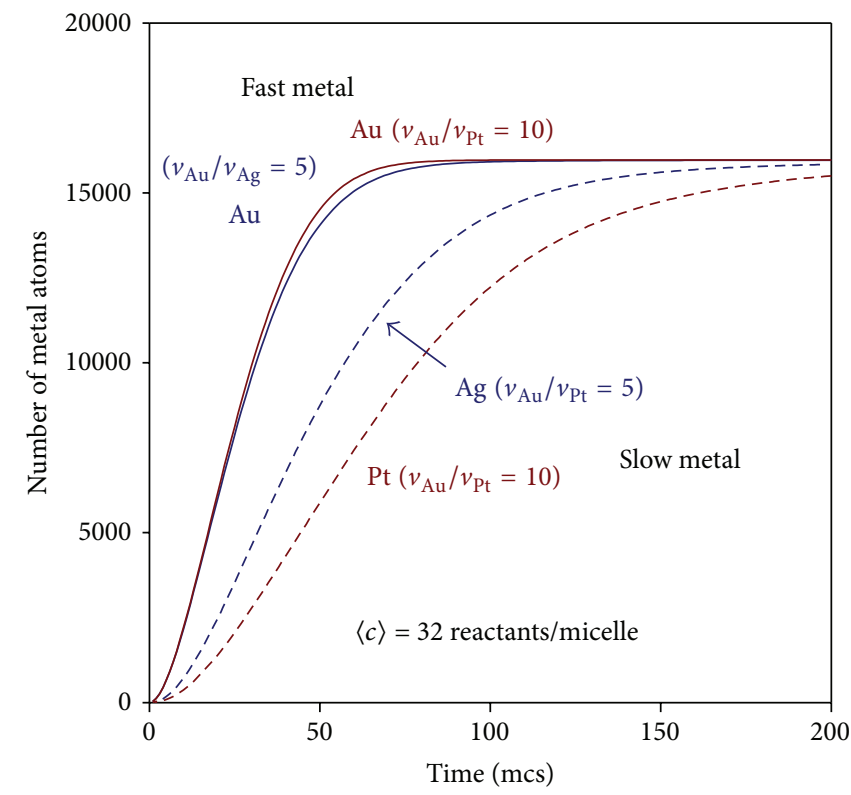

(a)

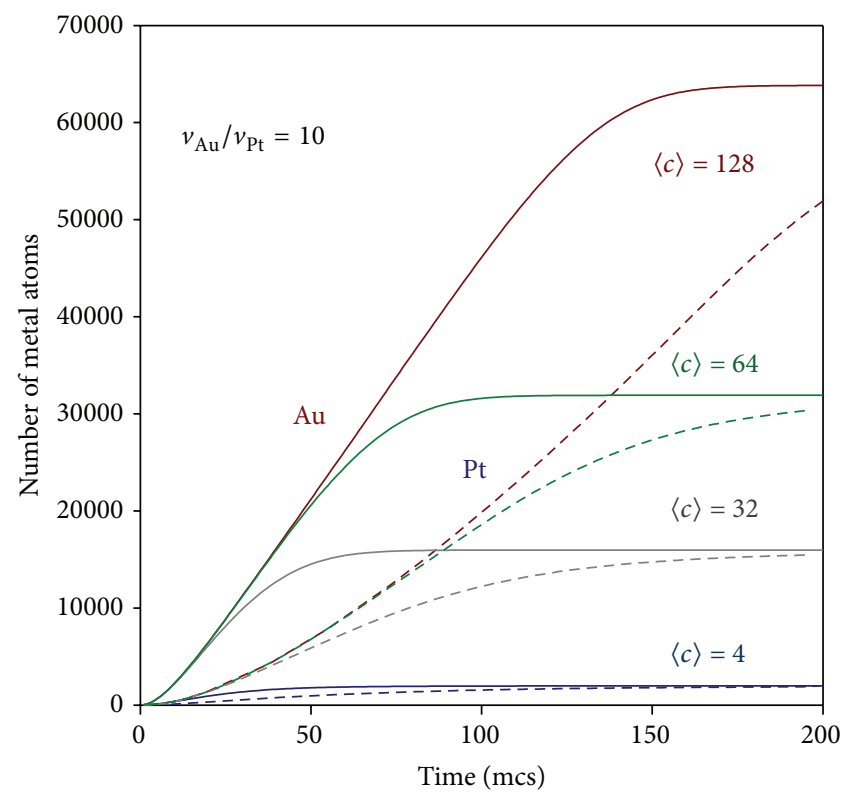

(b)

Figure 2: (a) Time evolution of the number of metal atoms obtained in micelles using different reduction rate ratio $v_{\text {fast }} / v_{\text {slow }}=5,10$. Continuous and discontinuous lines show the obtaining of fast ( $\mathrm{Au}$ ) and slow (Ag or Pt) metals, respectively. Synthesis conditions: flexible film $\left(k_{\mathrm{ex}}=5, f=30\right)$, average concentration $\langle c\rangle=32$ metal salts in a micelle. Scheme colour: blue lines represent $v_{\mathrm{Au}} / v_{\mathrm{Ag}}=5$; red lines represent $v_{\mathrm{Au}} / v_{\mathrm{Pt}}=10$. (b) Number of metal atoms obtained in micelles versus time using different concentration and keeping constant the reduction rate ratio $v_{\mathrm{Au}} / v_{\mathrm{Pt}}=10$.

forming the inner layers. That is, larger concentrations lead to more mixed cores (see Figure 1, from the top to the bottom). This would mean that the slow metal will be more accelerated by concentration compared to the faster one. Understanding this behaviour is not obvious. One must take into account that reverse micelles are demonstrated to be reaction medium that does not always obey classical assumptions. The dynamic properties of the microemulsion, which facilitates their use as confined reaction media, play a key role in the chemical kinetics. With the aim of explaining this unpredicted evolution of metal distribution in the nanostructure, a deeper kinetic study is explained below.

\subsection{Changing the Metal Salt Concentration: Kinetic Study.} The whole speed at which nanoparticle is built up depends not only on the chemical reduction rates but also on the intermicellar exchange rate and concentration. The final nanoparticle was proven to be due to the particular combination of these three factors, which in turn determine the particular sequence of deposition of the two metals on the growing particle [38]. The simulation model allows us to monitor the number of metal atoms produced in all micelles as the synthesis advances. Continuous lines in Figure 2(a) represent the number of faster atoms $(\mathrm{Au})$ and discontinuous lines correspond to slower ones (Ag, $v_{\mathrm{Au}} / v_{\mathrm{Ag}}=5$ or Pt, $v_{\mathrm{Au}} /$ $\left.v_{\mathrm{Pt}}=10\right)$. These data were obtained keeping fixed concentration $(\langle c\rangle=32$ atoms/micelle), that is, the synthesis conditions shown in Figures 1(c) and 1(d). The reduction rate ratios $v_{\mathrm{Au}} / v_{\mathrm{Ag}}=5$ and $v_{\mathrm{Au}} / v_{\mathrm{Pt}}=10$ are showed in blue and red, respectively. A plateau is reached when the reactants have been exhausted. As expected, the slower reduced metal is produced later (see discontinuous lines). Fast and slow curves appear more separately as the second metal reduction rate is slowing down (compare blue and red lines). Concerning the influence of concentration on the curves, Figure 2(b) shows the results obtained at different initial concentrations for a reduction rate ratio $v_{\mathrm{Au}} / v_{\mathrm{Pt}}=10$. As before, the quantities of $\mathrm{Au}$ and $\mathrm{Pt}$ produced at different concentrations as the synthesis advances are showed by continuous and discontinuous lines, respectively. One can observe that all Au curves have the same slope, except that concentration was very low. In this case, the reactant concentration appears to be the controlling factor, as expected. On the contrary, higher concentration does not result in a faster rate because the controlling step is the intermicellar exchange rate [47]. These data were obtained using an exchange parameter $k_{\mathrm{ex}}=5$, so only 5 molecules can be exchanged between micelles during each collision. That is, although the number of metal salts in one micelle was higher (e.g., $\langle c\rangle=32$ atoms/micelle), only a maximum of $5 \mathrm{Au}$ salts can be transferred in a collision. This implies that a maximum of $5 \mathrm{Au}$ atoms can be produced if there is enough reducing agent located in the micelle. This restriction also governs the exchange of slow metal salts, but, in this case, only $10 \%$ of the pairs of $\mathrm{Pt}$ salt and reducing agent carried by the same micelle react. The remaining reactants stay in the micelle, resulting in a local accumulation due to cage-like effect. Previous studies suggest that micelle plays as a cage $[37,47,50]$. The hypothesis is that reactants located inside the same micelle can fail to react the first time, but they have more opportunities as long as they remain in the same 


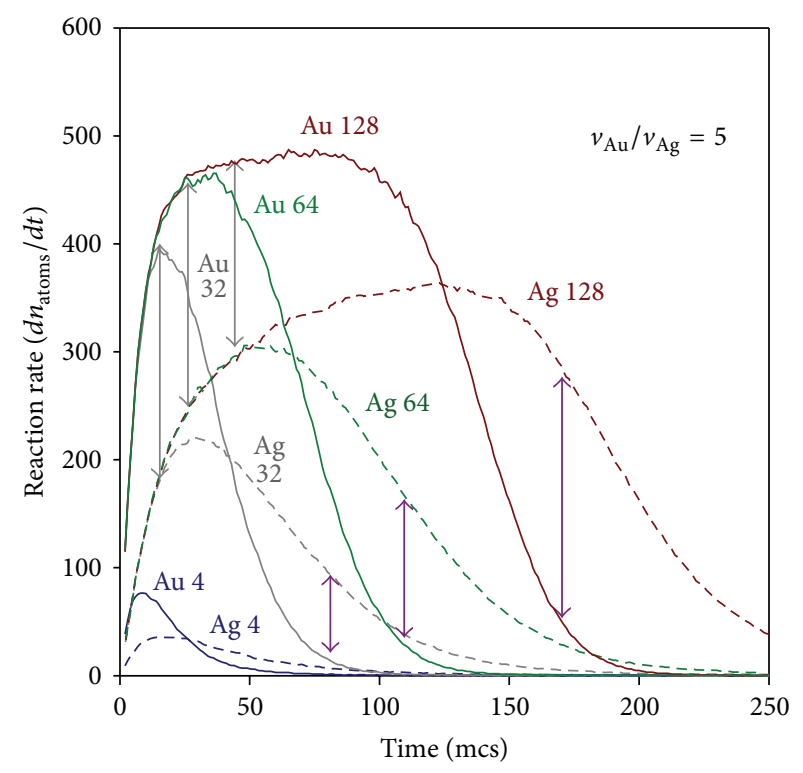

(a)

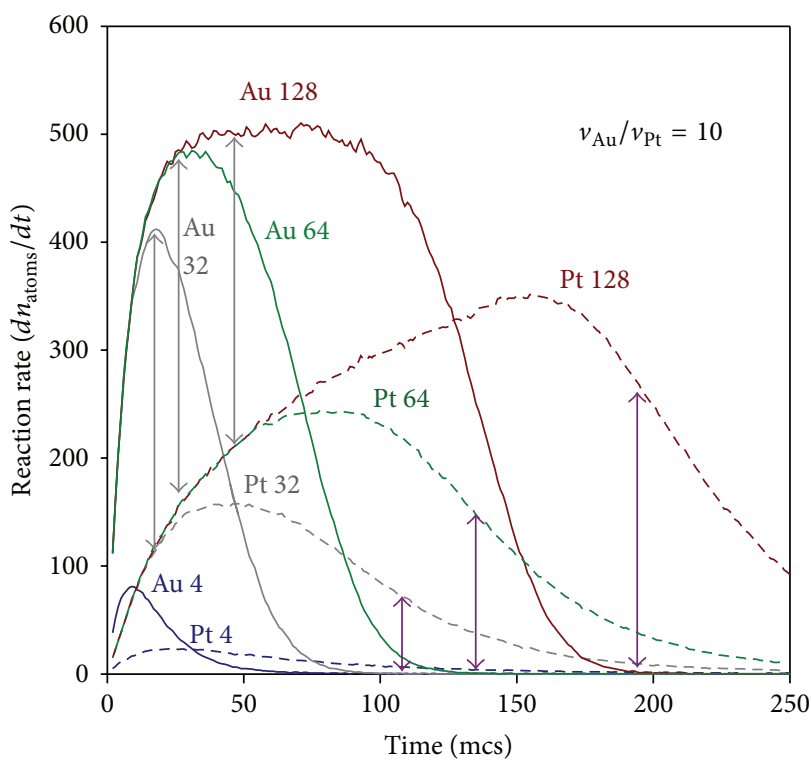

(b)

FIGURE 3: (a) Reaction rate versus time obtained using different reduction rate ratio: (a) $v_{\mathrm{Au}} / v_{\mathrm{Ag}}=5$ and (b) $v_{\mathrm{Au}} / v_{\mathrm{Pt}}=10$, and different average concentration in a micelle. Continuous and discontinuous lines show the obtaining of fast $(\mathrm{Au})$ and slow $(\mathrm{Ag}$ or Pt) metals, respectively. Synthesis conditions: flexible film $\left(k_{\mathrm{ex}}=5, f=30\right)$. Scheme colour: $\langle c\rangle=4,32,64$, and 128 metal salts in a micelle are represented by blue, grey, green, and red lines, respectively.

micelle. Consequently, the chemical reduction can continue independentally from a new intermicellar collision. In this way micelles allow for less encounters between reactants than continuous reaction media, but reactants confined in micelles remain near each other for much longer. As a result, slower metal reaction rate increases due to a cage-like effect.

The cage effect in micelles can be proved by calculating the overall reaction rate of each metal from the slopes of the curves showed in Figure 2. The overall reaction rate, estimated as $d n_{\text {atom }} / d t$, takes into account not only the chemical reduction rate but also the material intermicellar exchange rate. The calculated $\mathrm{Au}$ and $\mathrm{Pt}$ reaction rates are showed in Figure 3 as continuous and discontinuous lines, respectively, keeping the same scheme color compared to that used in Figure 2(b). All curves show a maximum, from which the usual decay is obtained. The growing rate before the maximum is due to the reactants redistribution between micelles, because only when the metal salt and the reducing agent are carried by the same micelle, chemical reduction can take place. That is, as the reactants are redistributed, more intermicellar collisions can result in chemical reaction, so reaction rate increases. After the maximum, the usual decay versus time can be observed as reactants have been consumed. In spite of the similarities, the fast and the slow metals show different behaviour. In relation to $\mathrm{Au}$, the fact that reactants are trapped inside micelles causes the $\mathrm{Au}$ reduction to be mainly controlled by the intermicellar exchange rate. This is reflected in the slopes of Au curves, which are equal before and after the peak for all concentration values and reduction rate ratios (see continuous lines in Figure 2). That is, Au reaction rate steadily increases while material has been exchanged between micelles and steadily decreases while there are $\mathrm{Au}$ salts. When concentration is high enough, Au reaction rate reaches a threshold dictated by the intermicellar exchange rate from which it cannot increase anymore (see continuous red line). At this stage, $\mathrm{Au}$ reaction rate remains constant as long as the quantity of $\mathrm{Au}$ precursor is enough. It implies that the intuitive idea that larger concentration always gives rise to a faster reduction rate was proved to be not always true in reverse micelle [47]. Note that intermicellar control cannot be reached at low concentration because precursor salt is exhausted earlier.

Concerning slow metal reaction rates (see discontinuous lines in Figure 2), one could expect a behaviour similar to $\mathrm{Au}$ rate, because exchange restrictions are the same for both metals. Indeed, before the peak, the only effect of increasing concentration on the slow reaction is the obtaining of higher and later maxima. The maximum is higher because high concentration results in faster reaction rate, which is reached later because of intermicellar exchange restrictions. Nevertheless, the speed at which the slow rate falls after the peak is strongly dependent on concentration, contrary to $\mathrm{Au}$ reaction rate. This unexpected result can be explained by the effect of confinement on both metals: in relation to the faster metal, once the intermicellar exchange allows the location of both reactants enclosed in the same micelle, the reaction is very quick. But in the case of a slow reduction, the reactants which fail to react the first time remain in the micelle, so they will be close together for longer. They can react later, regardless of whether or not an intermicellar exchange takes place. It results in a faster slow reaction rate due to cage-like effect. It is important to point out that the fast reduction is not affected by the cage effect, because no reactants accumulation takes place. On the contrary, slow 
rate depends on the exchange rate (as the Au case) and, in addition, depends on the accumulation of reactants, which will be higher as concentration is higher. Given that cage effect accelerates the slow reaction, it also results in a quicker decay of the slow reaction rate as observed in Figure 3.

3.3. Chemical Kinetics and Metal Distribution. The reaction rate profiles allow us to understand the influence of concentration and reduction rate ratio on resulting metal distribution in bimetallic nanostructures. The gap between the faster and the smaller reaction rates at the initial stages of the synthesis, when the core is building up, is shown by the grey arrows in Figure 3. Because the time required to reach equilibrium depends on concentration and reduction rate ratios, grey arrows are located at the stage at which $20 \%$ of total metal atoms have been deposited (the core is completed), for comparison purposes. The fact that the length of grey arrows is shorter as concentration is larger is directly related to metal distribution in the core: the shorter the gap, the smaller the difference between the reaction rates of the two metals, so the core is more mixed, as shown in Figure 1. That is, the high degree of mixture in the core observed at high concentrations is accounted for the acceleration of slow metal rate. In each pair of curves the factors affecting the rate (concentration, reduction rate ratio, and intermicellar exchange rate) are kept fixed, so the slow metal acceleration can be associated with the cage effect, which is more pronounced as concentration increases. By comparing Figures 3(a) and 3(b), one can observe that the gap is larger when $v_{\text {fast }} / v_{\text {slow }}$ ratio is higher, as expected. It results in a larger Au-enrichment in core, as observed in the structures shown in Figure 1.

Finally, the formation of the shell can also be explained on the basis of the differences between both rates. Pink arrows in Figure 3 show the gap between the faster and slower metals rates at later stages of the synthesis $(90 \%$ of the total metals were deposited), that is, when the shell is forming. As expected, a larger $v_{\text {fast }} / v_{\text {slow }}$ ratio gives rise to a larger gap, directly related to better separated shell. Likewise, the fact that shell is better defined at high concentration is also accounted for by the increasing gap between both rates as concentration increases. A priori, one could think that the slow metal enriched shell, enhanced by higher concentration, is contrary to the acceleration caused by cage effect. Although slow metal acceleration favours earlier deposition, a high concentration also means that the available quantity of slow metal at later stage of the synthesis is higher. This delay in slow metal reduction determines the pure shell formation observed at high concentration (see Figure 1).

These results can be extended to oil in water micelles (e.g., in the preparation of hydrophobic particles solved inside micelles) because the main cause of the cage effect is the confinement of reactants inside micelles, which takes place independently of the kind of micelle.

\section{Conclusions}

The fact that reactants are trapped into micelles plays a key role in metal arrangement in bimetallic nanocatalysts, because it affects the metals reduction in different ways. On the one hand, the intermicellar exchange rate is the controlling step of the fast metal reaction. On the other hand, slow metal reduction is also affected by the cage effect, which is more pronounced as concentration increases. It is reflected in the metal distribution of the nanocatalyst, which shows a more mixed core and a better defined shell as concentration is higher.

In summary, the surface composition of bimetallic nanocatalysts can be fine-tuned by choosing the adequate components of the microemulsion and reactant concentration. For a given couple of metals in a fixed microemulsion, the metal distribution of the final nanoparticle can be manipulated by changing the reactant concentration.

\section{Conflict of Interests}

The authors have no conflict of interests.

\section{Acknowledgments}

Works were supported by Ministerio de Ciencia e Innovación, Spain (MAT2012-36754-C02-01), and Xunta de Galicia (GRC2013-044, FEDER Funds, REDES 2014/019).

\section{References}

[1] R. Ferrando, J. Jellinek, and R. L. Johnston, "Nanoalloys: from theory to applications of alloy clusters and nanoparticles," Chemical Reviews, vol. 108, no. 3, pp. 845-910, 2008.

[2] S. Sun, C. B. Murray, D. Weller, L. Folks, and A. Moser, "Monodisperse FePt nanoparticles and ferromagnetic FePt nanocrystal superlattices," Science, vol. 287, no. 5460, pp. 1989$1992,2000$.

[3] A. Habrioux, W. Vogel, M. Guinel et al., "Structural and electrochemical studies of Au-Pt nanoalloys," Physical Chemistry Chemical Physics, vol. 11, no. 18, pp. 3573-3579, 2009.

[4] P. Hernández-Fernández, S. Rojas, P. Ocón et al., "Influence of the preparation route of bimetallic Pt-Au nanoparticle electrocatalysts for the oxygen reduction reaction," The Journal of Physical Chemistry C, vol. 111, no. 7, pp. 2913-2923, 2007.

[5] M. Boutonnet, S. Lögdberg, and E. E. Svensson, "Recent developments in the application of nanoparticles prepared from w/o microemulsions in heterogeneous catalysis," Current Opinion in Colloid and Interface Science, vol. 13, no. 4, pp. 270-286, 2008.

[6] J. R. Kitchin, J. K. Nørskov, M. A. Barteau, and J. G. Chen, "Role of strain and ligand effects in the modification of the electronic and chemical properties of bimetallic surfaces," Physical Review Letters, vol. 93, no. 15, pp. 156801-156804, 2004.

[7] J. A. Rodriguez and D. W. Goodman, "The nature of the metalmetal bond in bimetallic surfaces," Science, vol. 257, no. 5072, pp. 897-903, 1992.

[8] J. Suntivich, Z. Xu, C. E. Carlton et al., "Surface composition tuning of Au-Pt bimetallic nanoparticles for enhanced carbon monoxide and methanol electro-oxidation," Journal of the American Chemical Society, vol. 135, no. 21, pp. 7985-7991, 2013.

[9] R. Y. Parapat, V. Parwoto, M. Schwarze, B. Zhang, D. S. Su, and R. Schomäcker, "A new method to synthesize very active and stable supported metal Pt catalysts: thermo-destabilization of 
microemulsions," Journal of Materials Chemistry, vol. 22, no. 23, pp. 11605-11614, 2012.

[10] Z. Yin, D. Ma, and X. Bao, "Emulsion-assisted synthesis of monodisperse binary metal nanoparticles," Chemical Communications, vol. 46, no. 8, pp. 1344-1346, 2010.

[11] E. Westsson and G. J. M. Koper, "How to determine the coreshell nature in bimetallic catalyst particles?" Catalysts, vol. 4, no. 4, pp. 375-396, 2014.

[12] H. Zhang and N. Toshima, "Synthesis of Au/Pt bimetallic nanoparticles with a Pt-rich shell and their high catalytic activities for aerobic glucose oxidation," Journal of Colloid and Interface Science, vol. 394, pp. 166-176, 2013.

[13] H.-P. Liang, T. G. J. Jones, N. S. Lawrence, L. Jiang, and J. S. Barnard, "Understanding the role of nanoparticle synthesis on their underlying electrocatalytic activity," Journal of Physical Chemistry C, vol. 112, no. 11, pp. 4327-4332, 2008.

[14] M. A. López-Quintela, C. Tojo, M. C. Blanco, L. García Rio, and J. R. Leis, "Microemulsion dynamics and reactions in microemulsions," Current Opinion in Colloid and Interface Science, vol. 9, no. 3-4, pp. 264-278, 2004.

[15] M.-P. Pileni, "The role of soft colloidal templates in controlling the size and shape of inorganic nanocrystals," Nature Materials, vol. 2, no. 3, pp. 145-150, 2003.

[16] P. D. I. Fletcher, A. M. Howe, and B. H. Robinson, "The kinetics of solubilisate exchange between water droplets of a water-inoil microemulsion," Journal of the Chemical Society, Faraday Transactions, vol. 83, pp. 985-1006, 1987.

[17] K. Holmberg, "Surfactant-templated nanomaterials synthesis," Journal of Colloid and Interface Science, vol. 274, no. 2, pp. 355364, 2004.

[18] P. D. I. Fletcher, B. H. Robinson, F. Bermejo-Barrera, and D. G. Oakenfull, Microemulsions, Plenum Press, New York, NY, USA, 1982.

[19] R. Touroude, P. Girard, G. Maire, J. Kizling, M. BoutonnetKizling, and P. Stenius, "Preparation of colloidal platinum/ palladium alloy particles from non-ionic microemulsions: characterization and catalytic behaviour," Colloids and Surfaces A, vol. 67, pp. 9-19, 1992.

[20] M. Yashima, L. K. L. Falk, A. E. C. Palmqvist, and K. Holmberg, "Structure and catalytic properties of nanosized alumina supported platinum and palladium particles synthesized by reaction in microemulsion," Journal of Colloid and Interface Science, vol. 268, no. 2, pp. 348-356, 2003.

[21] C.-H. Chen, B.-J. Hwang, G.-R. Wang et al., "Nucleation and growth mechanism of Pd/Pt bimetallic clusters in sodium Bis(2ethylhexyl)sulfosuccinate (AOT) reverse micelles as studied by in situ X-ray absorption spectroscopy," Journal of Physical Chemistry B, vol. 109, no. 46, pp. 21566-21575, 2005.

[22] L. M. Magno, W. Sigle, P. A. Van Aken, D. G. Angelescu, and C. Stubenrauch, "Microemulsions as reaction media for the synthesis of bimetallic nanoparticles: size and composition of particles," Chemistry of Materials, vol. 22, no. 23, pp. 6263-6271, 2010.

[23] D. Buceta, C. Tojo, M. Vukmirovik, F. L. Deepak, and M. A. López-Quintela, "Controlling bimetallic nanostructures by the microemulsion method with sub-nanometer resolution using a prediction model," Langmuir, vol. 31, no. 27, pp. 7435-7439, 2015.

[24] J. Feng and C.-P. Zhang, "Preparation of $\mathrm{Cu}-\mathrm{Ni}$ alloy nanocrystallites in water-in-oil microemulsions," Journal of Colloid and Interface Science, vol. 293, no. 2, pp. 414-420, 2006.
[25] C. Tojo, M. de Dios, and M. A. López-Quintela, "On the structure of bimetallic nanoparticles synthesized in microemulsions," Journal of Physical Chemistry C, vol. 113, no. 44, pp. 19145-19154, 2009.

[26] D. G. Angelescu, L. M. Magno, and C. Stubenrauch, "Monte Carlo simulation of the size and composition of bimetallic nanoparticles synthesized in water in oil microemulsions," The Journal of Physical Chemistry C, vol. 114, no. 50, pp. 2206922078, 2010.

[27] S. Schwamborn, L. Stoica, and W. Schuhmann, "Activation/inhibition effects during the coelectrodeposition of PtAg nanoparticles: application for ORR in alkaline media," ChemPhysChem, vol. 12, no. 9, pp. 1741-1746, 2011.

[28] E. V. Abkhalimov and B. G. Ershov, "Pt ${ }_{\text {core }} \mathrm{Ag}_{\text {shell }}$ nanoparticlecatalyzed reduction of methylviologene with hydrogen in aqueous solution," Colloid Journal, vol. 72, no. 4, pp. 441-445, 2010.

[29] F. L. Deepak, G. Casillas-Garcia, R. Esparza, H. Barron, and M. Jose-Yacaman, "New insights into the structure of PdAu nanoparticles as revealed by aberration-corrected STEM," Journal of Crystal Growth, vol. 325, no. 1, pp. 60-67, 2011.

[30] T. Yonezawa and N. Toshima, "Mechanistic consideration of formation of polymer-protected nanoscopic bimetallic clusters," Journal of the Chemical Society, Faraday Transactions, vol. 91, no. 22, pp. 4111-4119, 1995.

[31] N. V. Long, T. D. Hien, T. Asaka, M. Ohtaki, and M. Nogami, "Synthesis and characterization of Pt-Pd alloy and core-shell bimetallic nanoparticles for direct methanol fuel cells (DMFCs): enhanced electrocatalytic properties of wellshaped core-shell morphologies and nanostructures," International Journal of Hydrogen Energy, vol. 36, no. 14, pp. 8478-8491, 2011.

[32] F. Tao, M. E. Grass, Y. Zhang et al., "Evolution of structure and chemistry of bimetallic nanoparticle catalysts under reaction conditions," Journal of the American Chemical Society, vol. 132, no. 25, pp. 8697-8703, 2010.

[33] F. Barroso and C. Tojo, "Modelling of nano-alloying and structural evolution of bimetallic core-shell nanoparticles obtained via the microemulsion route," Journal of Colloid and Interface Science, vol. 363, no. 1, pp. 73-83, 2011.

[34] F. Barroso and C. Tojo, "Designing bimetallic nanoparticle structures prepared from microemulsions," Journal of Physical Chemistry C, vol. 117, no. 34, pp. 17801-17813, 2013.

[35] J. Wang, H. F. M. Boelens, M. B. Thathagar, and G. Rothenberg, "In situ spectroscopic analysis of nanocluster formation," ChemPhysChem, vol. 5, no. 1, pp. 93-98, 2004.

[36] M.-L. Wu, D.-H. Chen, and T.-C. Huang, "Preparation of pd/pt bimetallic nanoparticles in water/AOT/isooctane microemulsions," Journal of Colloid and Interface Science, vol. 243, no. 1, pp. 102-108, 2001.

[37] O. F. Silva, M. A. Fernández, J. J. Silber, R. H. de Rossi, and N. M. Correa, "Inhibited phenol ionization in reverse micelles: confinement effect at the nanometer scale," ChemPhysChem, vol. 13, no. 1, pp. 124-130, 2012.

[38] C. Tojo, E. González, and N. Vila-Romeu, "The impact of the confinement of reactants on the metal distribution in bimetallic nanoparticles synthesized in reverse micelles," Beilstein Journal of Nanotechnology, vol. 5, pp. 1966-1979, 2014.

[39] P. Hernández-Fernández, S. Rojas, P. Ocón et al., "Influence of the preparation route of bimetallic Pt-Au nanoparticle electrocatalysts for the oxygen reduction reaction," Journal of Physical Chemistry C, vol. 111, no. 7, pp. 2913-2923, 2007. 
[40] B. N. Wanjala, J. Luo, R. Loukrakpam et al., "Nanoscale alloying, phase-segregation, and core-shell evolution of gold-platinum nanoparticles and their electrocatalytic effect on oxygen reduction reaction," Chemistry of Materials, vol. 22, no. 14, pp. 42824294, 2010.

[41] W. Zhang, L. Li, Y. Du, X. Wang, and P. Yang, "Gold/platinum bimetallic core/shell nanoparticles stabilized by a fréchet-type dendrimer: preparation and catalytic hydrogenations of phenylaldehydes and nitrobenzenes," Catalysis Letters, vol. 127, no. 34, pp. 429-436, 2009.

[42] J. Luo, P. N. Njoki, Y. Lin, D. Mott, L. Wang, and C.-J. Zhong, "Characterization of carbon-supported AuPt nanoparticles for electrocatalytic methanol oxidation reaction," Langmuir, vol. 22, no. 6, pp. 2892-2898, 2006.

[43] S. Tokonami, N. Morita, K. Takasaki, and N. Toshima, "Novel synthesis, structure, and oxidation catalysis of $\mathrm{Ag} / \mathrm{Au}$ bimetallic nanoparticles," Journal of Physical Chemistry C, vol. 114, no. 23, pp. 10336-10341, 2010.

[44] L. Feng, G. Gao, P. Huang et al., "Optical properties and catalytic activity of bimetallic gold-silver nanoparticles," Nano Biomedicine and Engineering, vol. 2, no. 4, pp. 258-267, 2010.

[45] J. Santhanalakshmi and P. Venkatesan, "Mono and bimetallic nanoparticles of gold, silver and palladium-catalyzed NADH oxidation-coupled reduction of Eosin-Y," Journal of Nanoparticle Research, vol. 13, no. 2, pp. 479-490, 2011.

[46] H. Zhang, J. Okuni, and N. Toshima, "One-pot synthesis of $\mathrm{Ag}-\mathrm{Au}$ bimetallic nanoparticles with $\mathrm{Au}$ shell and their high catalytic activity for aerobic glucose oxidation," Journal of Colloid and Interface Science, vol. 354, no. 1, pp. 131-138, 2011.

[47] C. Tojo, M. de Dios, D. Buceta, and M. A. López-Quintela, "Cage-like effect in Au-Pt nanoparticle synthesis in microemulsions: a simulation study," Physical Chemistry Chemical Physics, vol. 16, no. 36, pp. 19720-19731, 2014.

[48] S. Quintillán, C. Tojo, M. C. Blanco, and M. A. López-Quintela, "Effects of the intermicellar exchange on the size control of nanoparticles synthesized in microemulsions," Langmuir, vol. 17, no. 23, pp. 7251-7254, 2001.

[49] C. Tojo, M. C. Blanco, and M. A. López-Quintela, "Preparation of nanoparticles in microemulsions: a Monte Carlo study of the influence of the synthesis variable," Langmuir, vol. 13, no. 17, pp. 4527-4534, 1997.

[50] N. J. Turro and G. C. Weed, "Micellar systems as 'supercages' for reactions of geminate radical pairs. Magnetic effects," Journal of the American Chemical Society, vol. 105, no. 7, pp. 1861-1868, 1983. 

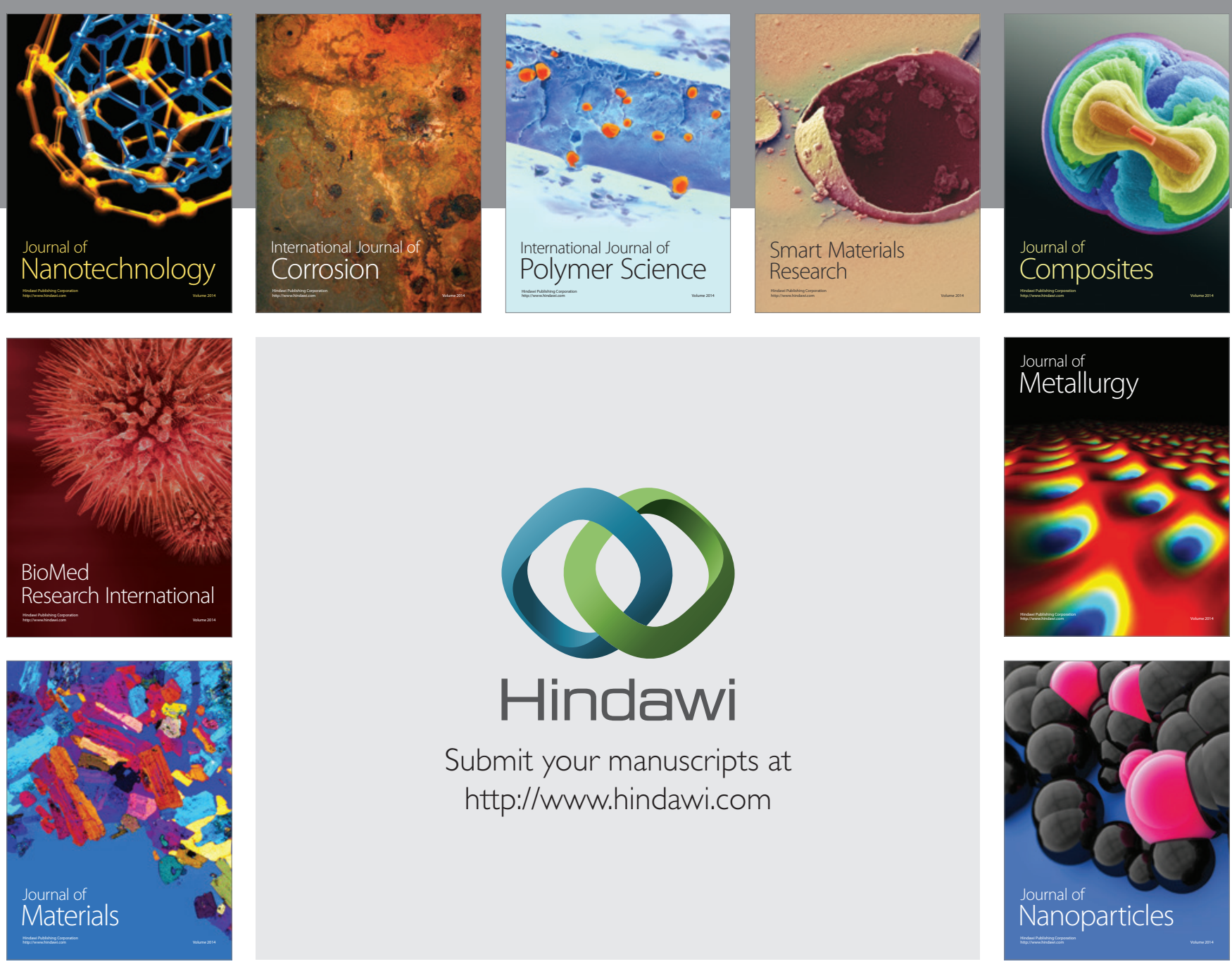

Submit your manuscripts at http://www.hindawi.com
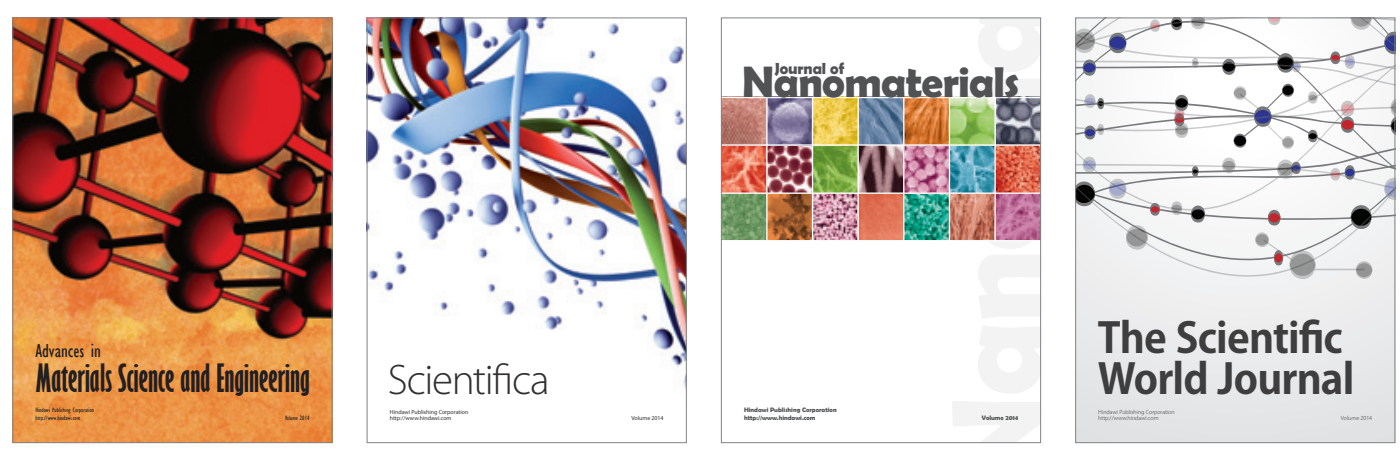

\section{The Scientific World Journal}
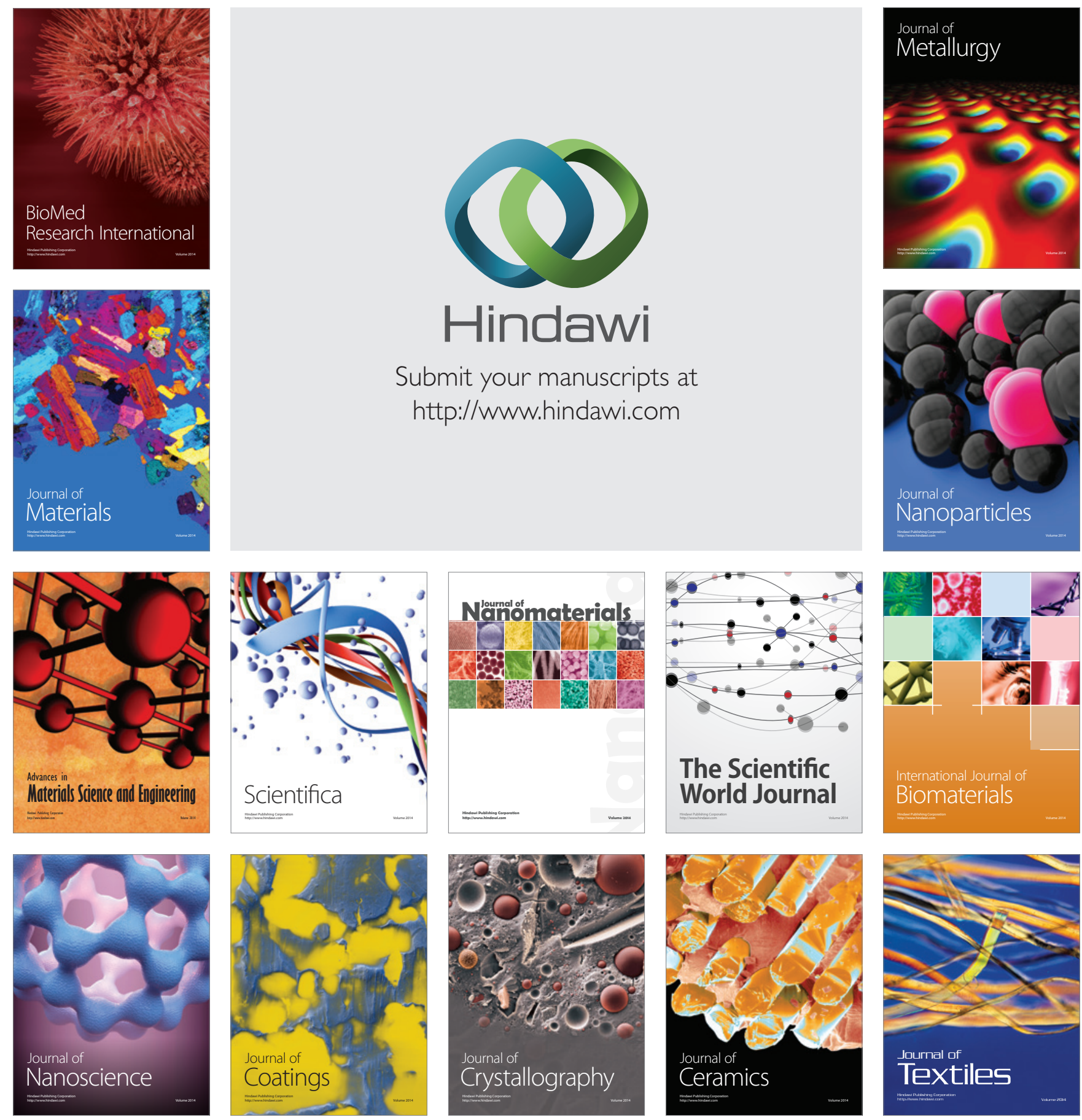